\title{
Using Electron Channeling Contrast Imaging for Misfit Dislocation Characterization in Heteroepitaxial III-V/Si Thin Films.
}

Julia Deitz ${ }^{1}$, Santino Carnevale ${ }^{2}$, Marc De Graef ${ }^{3}$, Yoosuf N. Picard ${ }^{3}$, Stephen A. Ringel ${ }^{2}$, Tyler Grassman $^{1,2}$, and David W. McComb ${ }^{1}$

1. Dept. of Materials Science \& Engineering, The Ohio State University, Columbus, OH, 43210, USA.

2. Dept. of Electrical \& Computer Engineering, The Ohio State University, Columbus, OH, 43210, USA.

3 Dept. of Materials Science \& Engineering, Carnegie Mellon University, Pittsburgh, PA, 15213, USA.

Characterization of extended defects is of paramount importance to a wide range of technologically important crystalline materials used in semiconductor devices. Imaging such defects in thin samples via (scanning) transmission electron microscopy, (S)TEM, provides critical insight into their structure and distribution. However, this is a fundamentally low-throughput approach (i.e., sample thinning, especially in plan-view, is time intensive) that can lead to significant delays in research and development cycles. Additionally, sample preparation artefacts can inadvertently impact the true nature of the sample (e.g., thinning can change the strain state of lattice-mismatched heteroepitaxial layers).

Electron channeling contrast imaging (ECCI), on the other hand, can be performed in a scanning electron microscope (SEM) and provides an alternative, high-throughput approach for imaging of extended defects. ECCI exploits changes in the back-scatter electron yield due to local displacement fields to yield information on defects in crystalline materials. In this contribution, we report our observations of misfit dislocations in samples of heteroepitaxial $\mathrm{GaP}$ on $\mathrm{Si}(001)$ substrates using ECCI. To the authors' knowledge, this is the first extensive application of this technique for imaging and analysis of misfit dislocations and their dynamics at the interface of two lattice-mismatched materials, as well as the first application to this particular material system. Epitaxial GaP on $\mathrm{Si}$ has been of interest for nearly four decades as a route for integration of III-V materials and devices with Si substrates and microelectronics. Nonetheless, only recently has substantial progress been made in overcoming issues related to epitaxy at the heterovalent interface (e.g. antiphase domains, stacking faults), with the successful development of both molecular beam epitaxy [1] and metalorganic chemical vapor deposition [2] based growth methodologies, opening possibilities for its use in the application of III-V/Si multijunction solar cells.

Figures 1 and 2 provide example data obtained through ECCI imaging in the backscatter geometry. The experiments were conducted on an FEI Sirion SEM using an accelerating voltage of $25 \mathrm{keV}$, a beam current of $\sim 0.5-3 \mathrm{nA}$, and a working distance of $5 \mathrm{~mm}$. A conventional solid-state annular BSE detector was used to capture all of the images shown here. Fig. 1A shows a section of an electron channeling pattern (ECP) taken across a range of tilt angles along the $<-110>$ band of a typical GaP/Si sample; the Kikuchi bands are identified in Fig. 1B. Fig. 1C shows ECCI micrographs of interfacial misfit dislocation segments from a $50 \mathrm{~nm}$ thick heteroepitaxial GaP layer on $\mathrm{Si}(001)$, recorded at the -220 channeling/diffraction condition, showing Burgers vector sensitivity due to the particular diffraction geometry (i.e. dark vs. light contrast). We will discuss results from a range of GaP/Si samples, including studies of $\mathrm{GaP}$ films covering a range of thicknesses (from $30-250 \mathrm{~nm}$ ), both as-grown and hightemperature annealed, for the analysis of misfit dislocation extension/glide at the strained interface (Figure 2). Overall, these results indicate that ECCI can produce results similar to TEM, making it an effective alternative for high-throughput materials characterization in this and similar material systems. 
We will augment our results with detailed image simulations for a range of electron channeling conditions.

\section{References:}

[1] T. J. Grassman et al, Appl. Phys. Lett. 94, 232106 (2009).

[2] T. J. Grassman et al, Appl. Phys. Lett. 102, 142102 (2013).

[3] The authors of this work acknowledge support from the Air Force Office of Scientific Research, MURI contract \# FA9550-12-1-0458, the Department of Energy under the FPACE program (DEEE0005398), and the Ohio Office of Technology Investment Third Frontier program.

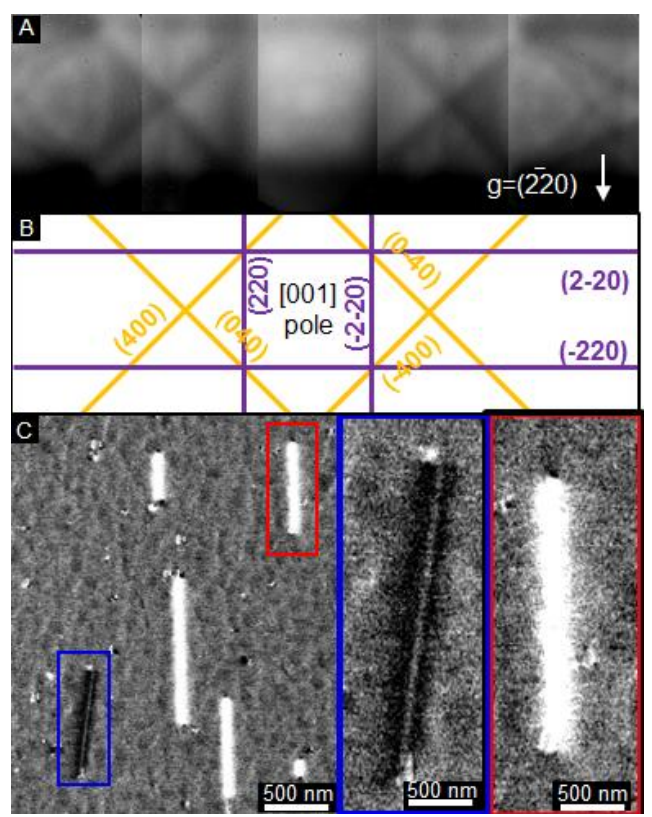

Figure 1.

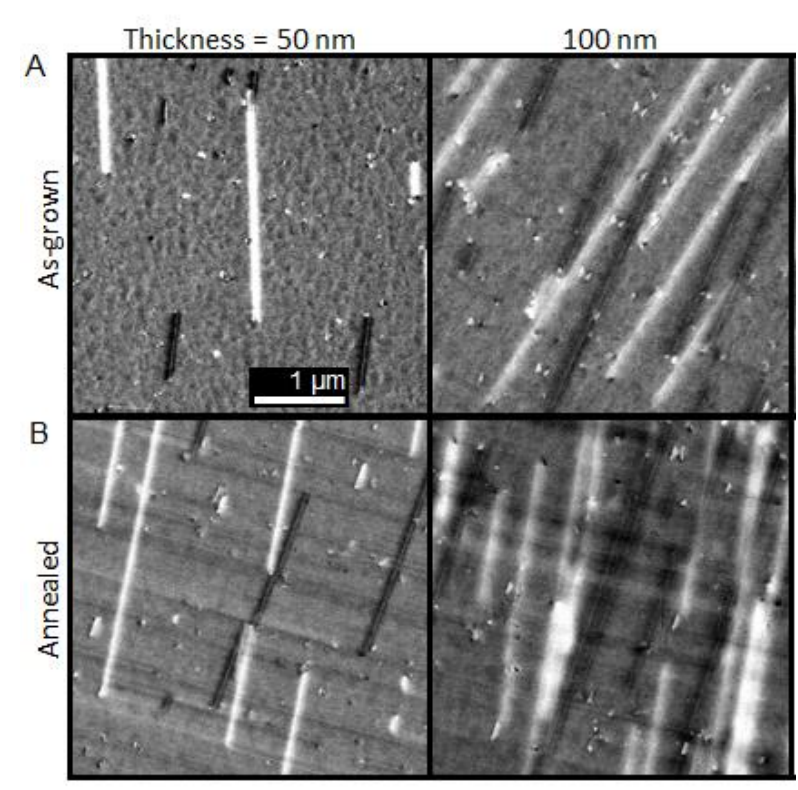

Figure 2.

Figure 1. (a) ECP of GaP (001) film with (b) electron channeling lines identified. ECCI micrographs recorded at $\mathrm{g}=-220$ showing (c) misfit dislocations (dark and light line features).

Figure 2. ECCI micrographs of GaP (001) films of varying thickness before and after annealing showing variations in misfit dislocation distributions. 